
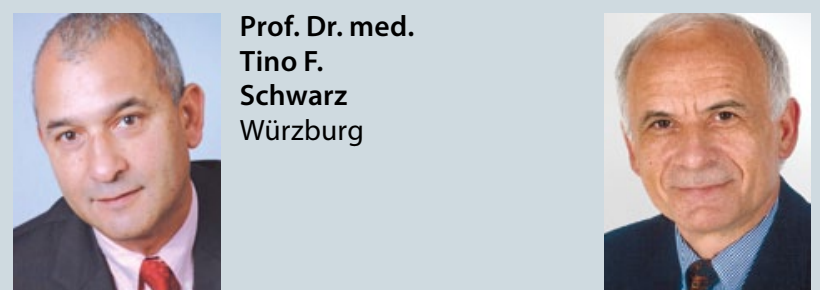

Prof. Dr. med.

Alfred Wirth

Bad Rothenfelde

\title{
Mehr späte Brustkrebsstadien bei jungen Frauen
}

\section{In den USA haben immer mehr Frauen unter 40 Jahren bei der Diagnose eines Mammakarzinoms bereits Fernmetastasen.}

$\mathrm{B}_{\mathrm{a}}^{\mathrm{r}}$ rustkrebs bei jungen Frauen verläuft aggressiver und geht mit schlechteren Überlebenschancen einher als bei älteren Frauen. Umso alarmierender sind aktuelle Daten aus den USA, wonach immer mehr Frauen zwischen 25 und 39 Jahren ein Mammakarzinom mit Fernmetastasen haben. Nach Daten aus den SEER-Registern des US National Cancer Institute hat ihre Zahl seit 1976 kontinuierlich zugenommen, von damals 1,53 pro 100.000 auf 2,90 pro 100.000 im Jahr 2009. Das entspricht einer jährlichen Steigerung um 2,07\%. „Der absolute Anstieg um 1,37 Fälle pro 100.000 Frauen in 34 Jahren ist zwar relativ gering, aber statistisch signifikant", schreiben die Autoren. Der Zuwachs an fernmetastasierten Mammakarzinomen war unabhängig von der ethnischen $\mathrm{Zu}$ gehörigkeit und dem urbanen oder ländlichen Wohnort. Die größte Steigerung wurde bei 25-34 Jährigen verzeichnet, von 0,81 Fällen pro $100.000 \mathrm{im} \mathrm{Jahr} 1976$ auf 2,14 Fälle 2009. Frauen mit Östrogenrezeptor-positiven Tumoren waren von der Zunahme ebenfalls stärker betroffen als Frauen mit Östrogenrezeptor-negativen. Unverändert geblieben ist dagegen die Inzidenz lokaler oder regionaler Brustkrebserkrankungen bei 25-39-jährigen Frauen. In anderen Altersgruppen wurde bei gar keinem Ausbreitungsstadium eine signifikante Zunahme festgestellt - mit einer Ausnahme: Bei den 40- bis 54-Jährigen wurden nach 1980 vermehrt lokal begrenzte Mammakarzinome entdeckt; die- ser Anstieg ist aber der Einführung des Mammografie-Screenings zu verdanken und beschränkt sich auf die Zeit bis 1990 .

Weshalb Mammakarzinome mit Fernabsiedelungen bei jungen Frauen zugenommen haben, ist laut Autoren unklar. Sie gehen jedoch davon aus, dass es „wahrscheinlich mehr als einen Grund" für diese Entwicklung gibt. Eine „stage migration“, eine Verschiebung durch Veränderungen in der Klassifikation und/oder Diagnostik schließen sie als (alleinige) Ursache aber aus. Frauen zwischen 25 und 39 mit lokoregionaler Erkrankung hatten in den Jahren 2000 bis 2004 eine 5-Jahres-Überlebensrate von $86,8 \%$, bei gleichaltrigen Frauen mit Fernmetastasen betrug sie nur $31,4 \%$.

Dr. Beate Schumacher

Johnson RH et al. Incidence of Breast Cancer With Distant Involvement Among Women in the United States, 1976 to 2009. JAMA

2013;309(8): 800-5; doi: 10.1001/jama.2013.776

\section{Akupunktur in der palliativen Krebsbehandlung?}

\section{Taugt die Akupunktur zur palliativen Krebsbehandlung? Diese Frage sollte ein} systematisches Review aller Studien zu diesem Thema beantworten.

nsgesamt fanden die Autoren 41 Studien zu folgenden Indikationen: Schmerz, Übelkeit, Hitzewallungen, Müdigkeit, Xerostoma, postoperativer Ileus, Angst und Schlafstörungen. Bei Weitem die meisten Studien beschäftigten sich mit Chemotherapie-bedingter Übelkeit; viele davon waren jedoch von methodisch fragwürdiger Qualität.

$\mathrm{Zu}$ den anderen Symptomen von Krebskranken gab es deutlich weniger Studien, die zudem häufig methodisch angreifbar waren. Die Autoren kommen dementsprechend $\mathrm{zu}$ einem ernüchternden
Schluss: Bei Übelkeit ist die Datenlage positiv, aber letztlich nicht völlig überzeugend. Bei allen anderen Symptomen von Krebspatienten ist die palliative Wirksamkeit der Akupunktur nicht belegt.

M. K. Garcia et al. Systematic review of acupuncture in cancer care: a synthesis of the evidence. J. Clin. Oncol. 2013 Mar 1;31(7):952-60. doi: 10.1200/JCO.2012.43.5818

Kommentar: In den vielen Krebsambulanzen wird die Akupunktur heute als ein Standard-Palliativum für die meisten Beschwerden von Krebspatienten eingesetzt.
Die vorliegende, sehr gut gemachte Analyse zeigt, dass dieses Vorgehen mehr auf Glauben als auf Evidenz beruht. Krebspatienten nehmen ganz offenbar diese Therapieform sehr gut an, vielleicht nicht zuletzt weil sie so gut wie keine ernsten Nebenwirkungen hat. Als kritische Ärzte sollten wir uns dennoch fragen, inwieweit die Beliebtheit und der scheinbare Nutzen der Akupunktur auf der Erwartungshaltung der Patienten und inwieweit auf belegbarer Effektivität beruhen. Einige werden sicher einwenden, dass dies einerlei sei - Hauptsache es hilft! Wer heilt hat Recht! Dem würde ich entgegenhalten, dass wir uns mit dieser Einstellung den Zugang zu der jeweils effektivsten Therapie erschweren - und das kann doch sicher nicht im Interesse unserer Patienten sein.

Prof. Edzard Ernst 